\title{
EFFECT OF HUMIDITY IN ETHYL CELLULOSE (EC) THERMELECTRET
}

\author{
M. S. GAUR*, RAMLAL, PRASHANT SHUKLA AND P. K. KHARE \\ Department of Physics, Hindustan College of Science and Technology, Farah (Mathura) U. P. India \\ 1. Department of Postgraduate study and Research in Physics, Rani Durgavati \\ University, Jabalpur (M. P.) India \\ (Received: 3 July 2007 - Accepted: 7 December 2007)
}

\begin{abstract}
The ethyl cellulose samples of thickness $30 \mu \mathrm{m}$ were prepared using solution grown method. The samples were polarized by means of thermal polarization for preparation of thermoelectret. The charge storage stability and the transport of detrapped charges in the EC thermoelectre were investigated by surface potential decay and open-circuit thermally stimulated discharge current (TSDC) analysis. The mean penetration depth for EC at different environment humidity and polarizing temperature were calculated. The results indicated that the charge stability of EC was significantly affected by relative humidity.
\end{abstract}

Keywords: ethyl cellulose, thermoelectret, charge storage stability, surface charge potential

\section{INTRODUCTION}

Ethyl cellulose is a weakly polar polymer having several applications not only in the field of medical material but also used as a Pharmaceutical material. The slight polar nature of the polymer is due to the difference in electronegativity of the main chain bonds and its side group. A charged piece (i.e. electret) of EC can be used as skin permeation enhancer. The stability of dipole orientation and space charge storage of EC is directly related to the effect of drug skin permeation. Therefore, the study of electret state, stability of dipole orientation and ability of space charge storage of EC for electret-drug transdermal delivery system are of great importance in basic research ${ }^{1-3}$. The objective of present work to investigate the effect of environment humidity and heat treatment on charge storage stability and transport of detrapping charge for EC thermoelectret by surface potential decay and open circuit thermally stimulated discharge current (TSDC) techniques.

\section{EXPERIMENTAL DETAILS}

The ethyl cellulose of molecular weight 40000, density 1.07-1.18, ethoxyl percentage of $44-48 \%$, viscosity level 25 (specific range 20-29) was supplied by Loba Chemical Ltd, Bombay (India). All samples were prepared by using the solution grown technique. The solution of EC was prepared in a glass beaker by dissolving EC (5g) in $100 \mathrm{~mL}$ Benzene (analytical grade Merck). The solution was then kept for $10 \mathrm{~h}$ to become homogeneous and transparent. The solution thus prepared was poured onto an optically plane glass plate floating on mercury pool and the solvent was then allowed to evaporate inside an oven at room temperature for $24 \mathrm{~h}$ to yield desired samples. The dried samples annealed at $50^{\circ} \mathrm{C}$ in oven for 6 hours in order to remove residual solvent. One surface of the samples were vacuum aluminized over central circular area of diameter $3.5 \mathrm{~cm}$. Samples, after vacuum aluminization were thermally polarized at different polarizing field and temperature conditions. The polarized sample was mounted in a electrode assembly with the nonmetallized surface parallel to sensing electrode at a distance of $2 \mathrm{~mm}$ for measurement of TSDC in open circuit mode. TSDC is recorded by means of keithley electrometer $610 \mathrm{C}$ with heating rate of $3^{\circ} \mathrm{C} / \mathrm{min}$. The surface potential was measured using capacitive probe method ${ }^{3-4}$.

\section{Results and Discussion}

It is observed that charge storage in EC thermoelectret in air is a function of environment humidity after thermal charging at $65^{\circ} \mathrm{C}$ with polarizing field of $200 \mathrm{kV} / \mathrm{cm}$. The surface potential decreases, when the samples were stored at different humidity condition for $240 \mathrm{~min}$. (Fig.1). Fig. 1 shows the surface potential decay curves of EC samples stored under different environment humidity before thermal charging at $65^{\circ} \mathrm{C}$ (i.e. below $\mathrm{T}$ ). The polar polymers are quite sensitive with humidity because of the polar group attached in the main chain. In presence of polar group the oxygen in carbonyl group can easily react with the $\mathrm{H}^{+}$in water, which leads to the absorption of water near the surface and in the bulk, the increase of the conductivity of the surface and bulk ${ }^{1}$ and the enhancement of charge decay corresponding to decay in potential (fig.1).

$$
\mathrm{I}=\mathrm{I}_{0} \exp [-\mathrm{A} / \mathrm{kT}]=\mathrm{n} \alpha_{0} \exp [-\mathrm{A} / \mathrm{kT}]
$$

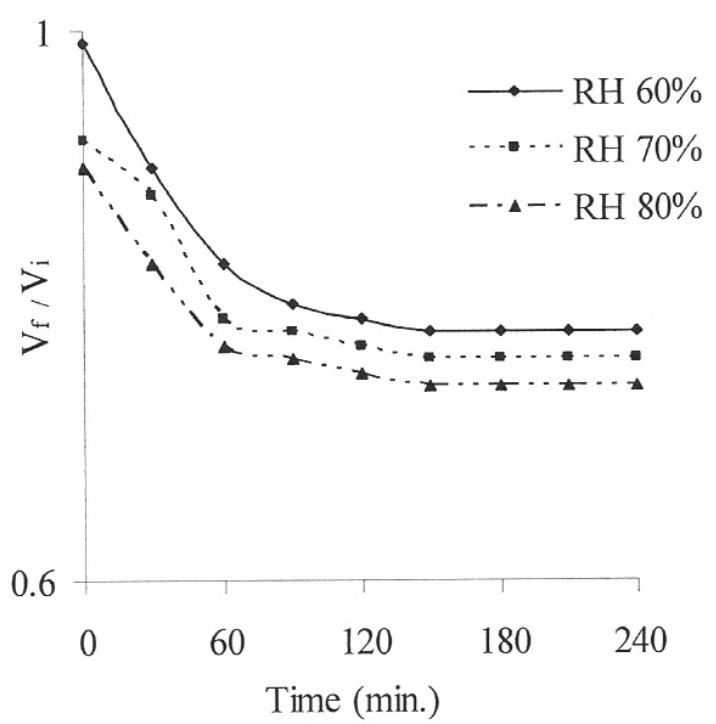

Fig.1: Surface potential decay characteristics of EC thermoelectret polarized at $65^{\circ} \mathrm{C}$ with polarizing field of $200 \mathrm{kV} / \mathrm{cm}$ under different $\mathrm{RH}$

Fig.2 depicts the TSDC spectra of EC after polarizing at room temperature under different environment humidity. The TSDC spectra appeared one $\beta$ peak near $50^{\circ} \mathrm{C}$ and one $\alpha$ peak near $100^{\circ} \mathrm{C}$. With increasing moisture content, the $\beta$ peak temperature shifted obviously toward lower value, while the position of $\alpha$ peak remains the same. The activation energies of $\beta$ and $\alpha$ peak is evaluated using initial rise method. The TSDC process follows first order kinetics, if detrapping of charge carriers is assumed to be negligible:

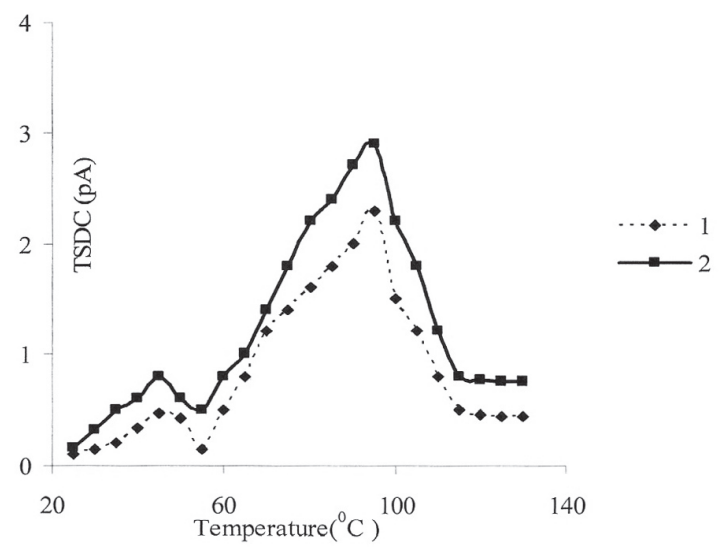

Fig.2: TSDC spectra of EC thermoelectret polarized at Rt with polarizing field of $200 \mathrm{kV} / \mathrm{cm}$ under different RH (i.e. $1-60 \%, 2-80 \%$ ) 


$$
\mathrm{I}=\mathrm{I}_{0} \exp [-\mathrm{A} / \mathrm{kT}]=\mathrm{n} \alpha_{0} \exp [-\mathrm{A} / \mathrm{kT}]
$$

where $\mathrm{I}$ is the current intensity, $\mathrm{n}$ the concentration of trapped charge carriers, $\alpha_{0}$ the escape frequency, $\mathrm{k}$ the Boltzman constant, $\mathrm{T}$ the temperature and $\mathrm{A}$ is activation energy. When the TSDC experiment carried out at $\mathrm{RH}=60 \%$ (i. e. it means that sample was subject to 240 min under the influence of RH $60 \%$ before polarization), the activation energies of peak $\beta$ and $\alpha$ peak are $0.37 \mathrm{eV}$ and $0.62 \mathrm{eV}$, but $0.31 \mathrm{eV}$ and $0.58 \mathrm{eV}$ at $\mathrm{RH}=80 \%$, respectively. It shows the activation energy is highly affected by environment humidity. Fig.2 indicates that the higher the environment humidity, the larger the area of curve, because the water molecule entering into crystalline or noncrystalline sites of polymer are supposed to be promoted the trapping of charge carriers. The water molecules are electron receptor. The formation of hydrogen bond by water molecules and macromolecules reduces the mutual attraction between polymer molecules and activation energy of dipole orientation, which is affecting a relaxation time of dipoles ${ }^{5-6}$. For the higher environment humidity, the larger number of water molecule absorbed near the surface and in the bulk of EC brought about the loss of charges or the depolarization of dipole in the thermoelectret and reducing the charge storage stability. The dependence of the mean penetration depth (r) on environment humidity is shown in Fig.3. The mean penetration depth is calculated using following relation ${ }^{7}$ :

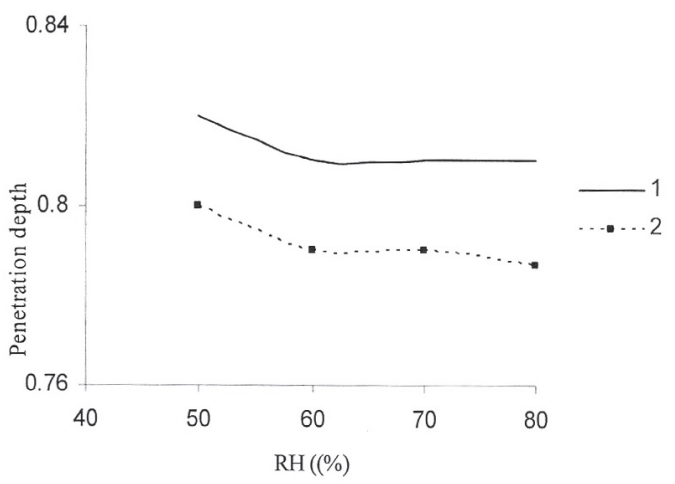

Fig.3: Mean penetration depth versus RH characteristics (i. e. 1-Rt, 2$65^{\circ} \mathrm{C}$ ) for EC thermoelectret polarized with polarizing field of $200 \mathrm{kV} / \mathrm{cm}$

$$
\begin{gathered}
\mathrm{r}=\dot{\mathrm{r}} / \mathrm{L}=1 /(1+\sigma / \mathrm{Q}) \ldots \ldots \ldots \ldots \ldots \ldots \ldots \ldots \ldots \\
\text { where } \hat{\mathrm{r}}=\dot{\mathrm{r}}_{\mathrm{n}+}^{\prime} \mathrm{r}_{\mathrm{p}}
\end{gathered}
$$

where $\dot{r}$ is the total depth, $\dot{r}_{n}$ mean depth of injected charge, $\dot{r}_{p}$ mean depth of compensating charge, $\sigma$ surface charge density and $Q$ charge released. It has been observed that penetration depth decreases with increase in relative humidity.

The mean penetration depth is very small, if samples (i.e. $\mathrm{RH}=60 \%$ ) are polarized at room temperature $(\mathrm{R})$. The mean penetration depth increases with increase in environment humidity (from $\mathrm{RH}=60 \%$ to $\mathrm{RH}=80 \%$ ) and it is shifted probably to the back of the electrode. As stated above, a large amount of water molecules was absorbed into the polymer when subjected to high humidity. Thus, water molecules could move easily in the bulk of EC samples at Rt. These water molecules may be act as the carriers of detrapping charges that shifted towards the back electrode under the internal electric field of the thermoelectret. The water molecules are supposed to be providing additional charge carrier and increases the concentration of the carriers and changes the geometric distributions of deposited charges in the sample. Therefore, the increase of RH could promote the shift of mean penetration depth towards the back electrode. Fig. 3 depicts the effect of different environmental humidity on mean penetration depth for thermally polarized EC.

Fig.4 is representing the TSDC spectra for EC samples $(\mathrm{RH}=70 \%)$ at different polarizing temperatures. When the TSDC was recorded for samples polarized at room temperature (i. e. curve 1) shows two peaks, one at low temperature was around $50^{\circ} \mathrm{C}(\beta$ peak) and the other at higher temperature peak at about $90^{\circ} \mathrm{C}$ ( $\alpha$ peak). As the temperature rose near T during TSDC, the dipole orientation, caused by polarizing field deposited on sample surface when charged at $\mathrm{R}_{t}$, led to the first peak $\left(\right.$ at $\left.50^{\circ} \mathrm{C}\right)$. The second peak of $90^{\circ} \mathrm{C}$ originated from the space charge. When the polarizing temperature increases, the two peaks shifted towards higher temperature, respectively, then become singular peak and the charge density reduced gradually (fig. 4). The $\alpha$ peak originated jointly from the space charge detrapping and dipole deorientation
${ }^{6-7}$. As the polarizing temperature increases the $\alpha$ peak is shifted towards high temperature may be due to relaxation frequency of the dipole and the space charge were not the same. The TSDC at different temperature (fig.4) may be caused by heat stimulating at high temperature which either restrained the charge from captured by shallow-trap, or because of activation, re-detrapped the charge which had already captured by shallow trap during polarization and recaptured some of these charges by deeper-trap. As compared with polarizing at $\mathrm{R}$, more charges were captured in deeper traps, which contributed to the increase in charge storage lifetime of EC after rising the polarizing temperature. It could increase the charge storage stability was further confirmed by both the curves of mean penetration depth $(\mathrm{r})$ and surface potential decay V(t)[fig.5]. It is suggested that the polymer shows stronger charge storage capability when polarized at $65^{\circ} \mathrm{C}$ than Rt. EC is a weakly polar material, the obvious shifts of mean penetration depth of sample due to the thermal polarization reduces the effect of surroundings humidity on charge storage life time and improve the charge storage stability of EC.

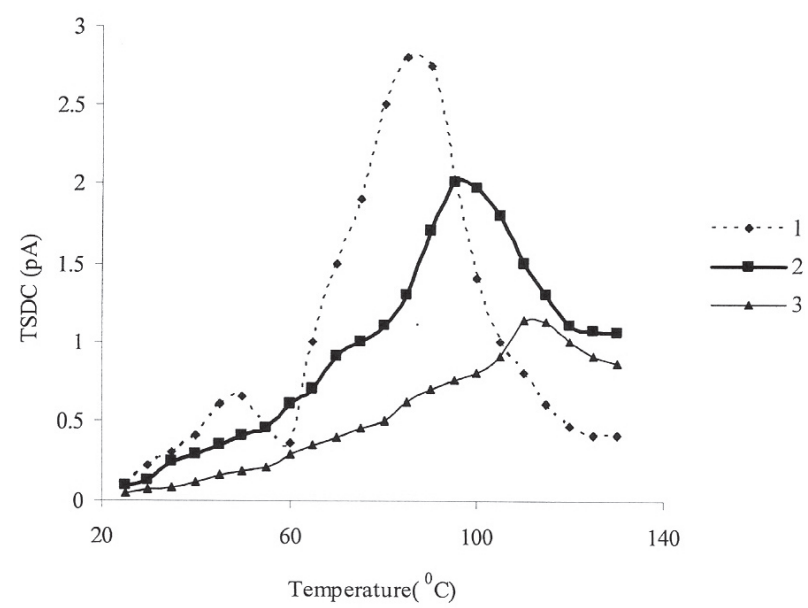

Fig.4: TSDC spectra of EC thermoelectret polarized at Rt, $50^{\circ} \mathrm{C}, 60^{\circ} \mathrm{C}$ with polarizing field of $200 \mathrm{kV} / \mathrm{cm}$ under $70 \% \mathrm{RH}$

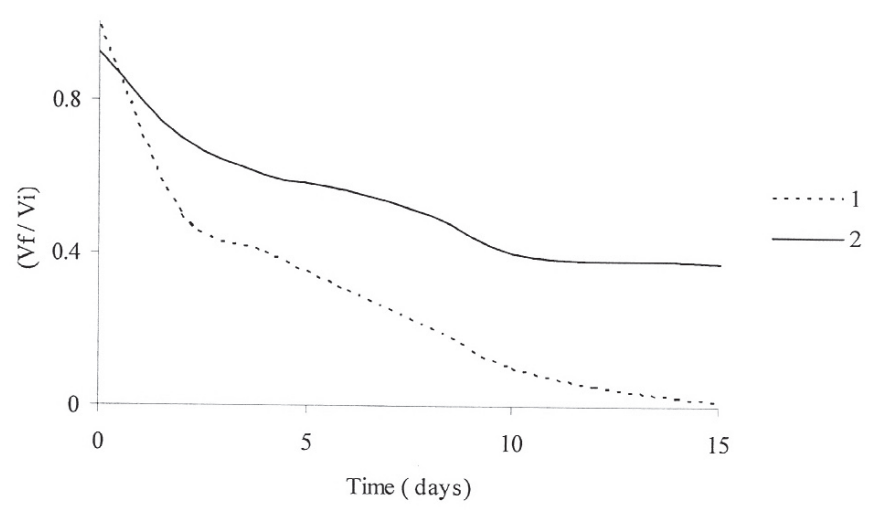

Fig. 5: Surface potential decay characteristics of EC thermoelectret at certain temperature $\left(1-65^{\circ} \mathrm{C}, 2-\mathrm{Rt}\right)$ with polarizing field of $200 \mathrm{kV} / \mathrm{cm}$ under $60 \% \mathrm{RH}$

\section{CONCLUSION}

The charge storage and transport in EC thermoelectret have been investigated using TSDC and surface potential measurement. These properties are strongly affected by polarizing temperature and environment humidity. The poor charge stability of EC thermoelectret has been observed under higher RH. However, polarizing temperature can significantly improve the space charge storage stability of EC. It is concluded that the interaction between dipoles and space charge under different humidity and polarizing condition reveals the suitability of EC thermoelectret for drug delivery system characterized by permeation enhancement. 


\section{ACKNOWLEDGEMENT}

Authors (M S Gaur and Prashant Shukla) gratefully acknowledge the financial support granted by Uttar Pradesh Council of Science and Technology, Lucknow (U. P.) India

\section{REFERENCES}

1.- C. L Jian Jiang, X. Zhongfu, C. Gangjin, W. Zhengzhong, J. Electro 123, 61, (1998)

2.- R. H. Mahat, D. A. Bradley, Y. M. Amin, C. Y. Wong, D. L. Su, Radiation Physics and Chemistry, 61, 469, (2001)
3.- G. M. Sessler eds. Electret vol.1 and 2, $3^{\text {rd }}$ edition Laplacian press, Margan hill, CA, 1999.

4.-. J. Van Turnhout, Advances in Static Electricity (W de Geest Auxxilia, Brussels), 1971

5.- P. K. Khare, J. M. Keller, M. S. Gaur, R. Singh, S. C. Datt, Polymer International 39, 303 (1996)

6.- L. Jinde, L. Deming, Physical principles and applications of dielectric materials, Zhongshan university publishing house, Guang Dong, 1992

7.- R. Singh R, J. M. Keller, S. C. Datt,. Ind. J. of Pure and Appl. Phys 30, 291 (1992 